\title{
THE MEAN LATTICE POINT DISCREPANCY
}

\author{
by M. N. HUXLEY
}

(Received 25th April 1994)

\begin{abstract}
Consider a sufficiently smooth simple closed convex plane curve enclosing the origin, expanding linearly with time. The root mean square of the discrepancy (number of lattice points minus area) from time $t=M$ to $t=M+1$ is almost as small as the root mean square discrepancy from time $t=0$ to $t=M$, so the discrepancy has no memory.
\end{abstract}

A.M.S. subject classification: $11 \mathrm{~K} 38,11 \mathrm{P} 21$.

Let $C$ be a simple closed convex curve in the plane, winding once round the origin, given by $x=x(t), y=y(t)$ with the functions $x(t), y(t)$ four times continuously differentiable, and with the radius of curvature bounded away from zero and infinity. Let $A$ be the area of $C$. For $M$ large and for $u, v$ in $0 \leqq u \leqq 1,0 \leqq v \leqq 1$, we consider the enlarged and shifted curve $C_{M}(u, v)$, the locus of the point $(M x(t)+u, M y(t)+v)$. Let $N_{M}(u, v)$ be the number of integer points inside $C_{M}(u, v)$, and let $D_{M}(u, v)$ be the signed discrepancy

$$
D_{M}(u, v)=N_{M}(u, v)-A M^{2}
$$

The root mean square value of $D_{M}(u, v)$ over $u$ and $v$ is bounded above and below by multiples of $\sqrt{ } M$ [9]. We consider the curve $C_{M}(0,0)$ for $M$ varying, and drop $u$ and $v$ from the notation, writing $C_{M}, N_{M}$ and $D_{M}$ for the curve $C_{M}(0,0)$, the number of lattice points $N_{M}(0,0)$ and the signed discrepancy $D_{M}(0,0)$. Nowak [10] showed that the root mean square of $D_{M}$ over $M$ is $O(\sqrt{ } M)$. He also showed [11] that $D_{M}(u, v)$ is not $O(\sqrt{ } M)$ uniformly in $u, v$, and $M$.

We give a localised form of Nowak's result, which shows that the discrepancy $D_{M}$ has no memory: if we imagine $M$ as a time variable, then $D_{M}$ returns almost to its mean square value within a bounded time. We note that when $M$ increases by more than $\sqrt{2}$, then every lattice square which was cut by the curve now lies completely inside it. As a corollary we get a pointwise bound for the discrepancy $D_{M}$ with the usual exponent $2 / 3$ $[1,2,3,5,12]$ which corresponds to the existence of the radius of curvature. We use Fourier theory, together with the duality of points and lines in plane projective geometry. This duality was also used in [7] in estimating the number of rational points with bounded denominator which lie close to the curve $C$. Relations between these 
problems, and others involving lattice points and plane curves, form the theme of a forthcoming book [8].

Theorem. Suppose that the curve $C$ encircles the origin, is four times differentiable, and has radius of curvature bounded away from zero and infinity. Then for $M$ large

$$
\int_{M}^{M+1}\left|D_{t}\right|^{2} d t=O(M \log M)
$$

The implied constant depends on the shape and size of $C$, and on its position and orientation with respect to the coordinate axes.

Corollary. Under the same conditions

$$
D_{M}=O\left(M^{2 / 3}(\log M)^{1 / 3}\right)
$$

We use the Fourier series discovered by Kendall [9], a remarkable extension of a formula for the discrepancy of an ellipse as an infinite series of Bessel functions first stated by Voronoi [13]. The Fourier coefficients correspond to short arcs of the curve where the gradient is close to a rational number. Hlawka [6] and Herz [4] have computed the Fourier coefficients to great accuracy for the n-dimensional lattice point problem.

Lemma 1 (Fourier series for the discrepancy). If there is no lattice point on $C_{M}(u, v)$, then the discrepancy $D_{M}(u, v)$ is equal to the sum of a Fourier series

$$
\sum_{h} \sum_{k} a_{M}(h, k) e(h u+k v) .
$$

The Fourier coefficients are given by

$$
a_{M}(0,0)=A M^{2}
$$

and for $h$ and $k$ not both zero

$$
a_{M}(h, k)=-b_{M}(k,-h)+\overline{b_{M}(-k, h)}+\varepsilon_{M}(h, k)
$$

where $b_{M}(\mathbf{v})$ is defined as follows. Let $P$ be the point of the curve $C$ where the vector $\mathbf{v}$ is in the anticlockwise direction of the tangent. Let $\rho$ be the radius of curvature of $C$ at $P$, and let $L(\mathrm{v})=\overrightarrow{O P} \times \mathrm{v}$ be the moment about the origin of $\mathrm{v}$ acting at $P$. Then

$$
b_{M}(\mathbf{v})=\frac{\sqrt{ }(M \rho)}{2 \pi i|\mathbf{v}|^{3 / 2}} \mathrm{e}\left(-M L(\mathbf{v})+\frac{1}{8}\right)
$$


The residual term satisfies

$$
\varepsilon_{M}(h, k)=O\left(M^{-1 / 2}\left(h^{2}+k^{2}\right)^{-5 / 4}\right) .
$$

Lemma 2 (mean to maximum). Let $\delta$ satisfy $0<\delta \leqq M / 2$. If $\left|D_{M}\right| \geqq 5 A \delta M$, then we have

and

$$
\left|\int_{M-\delta}^{M} \frac{D_{t}}{\sqrt{ } t} d t\right|+\left|\int_{M}^{M+\delta} \frac{D_{t}}{\sqrt{ } t} d t\right| \geqq \frac{\delta\left|D_{M}\right|}{\sqrt{ }(6 M)}
$$

$$
\int_{M-\delta}^{M+\delta} D_{t}^{2} d t \geqq \frac{\delta D_{M}^{2}}{4}
$$

Proof. Since $C_{t}$ lies outside $C_{u}$ for $t>u$, the number of lattice points caught inside $C_{t}$ is a non-decreasing function of $t$. For $t \geqq u$

$$
N_{\imath} \geqq N_{u}, D_{t} \geqq D_{u}-A\left(t^{2}-u^{2}\right) .
$$

Hence if $D_{M}>0$, then for $M \leqq t \leqq M+\delta$

$$
D_{t} \geqq D_{M}-A\left(t^{2}-M^{2}\right) \geqq D_{M}-A(t-M)(t+M) \geqq D_{M}-5 A \delta M / 2 \geqq D_{M} / 2,
$$

and similarly if $D_{M}<0$, then for $M-\delta \leqq t \leqq M$

$$
D_{\mathrm{t}} \leqq D_{M} / 2<0 \text {. }
$$

The results follow at once by integration over $t$.

Our next lemma uses the functions $\operatorname{sinc}^{2} x, \Lambda(s)$, where

$$
\operatorname{sinc} x=\frac{\sin \pi x}{\pi x}, \Lambda(s)=\max (1-|s|, 0) \text {. }
$$

These functions are both non-negative, with a maximum at the origin, and form a Fourier transform pair.

Lemma 3 (short interval means). Let $s_{1}, \ldots, s_{R}$ be real numbers, and let

$$
F(x)=\sum_{r=1}^{R} a_{r} \mathrm{e}\left(s_{r} x\right),
$$

where $a_{1}, \ldots, a_{R}$ are any coefficients. For positive real numbers $\delta$ and $\Delta$, and any real $X$, let

$$
I(\Delta)=\int_{x-\Delta}^{x+\Delta}|F(x)|^{2} d x
$$

and

$$
S(\delta)=\sum_{i} \sum_{j} a_{i} \overline{a_{j}} \mathrm{e}\left(s_{i} X-s_{j} X\right) \Lambda\left(\frac{s_{i}-s_{j}}{\delta}\right)
$$




$$
=\frac{1}{\delta} \int\left|\sum_{y \leqq s_{i} \leqq \delta} a_{i} \mathrm{e}\left(s_{i} X\right)\right|^{2} d y .
$$

Then $S(\delta)$ is positive, and

$$
I(\Delta) \leqq \frac{\pi^{2} \Delta}{2} S\left(\frac{1}{2 \Delta}\right)
$$

Proof. We take $X=0$. Since

$$
\operatorname{sinc} x=\frac{\sin \pi x}{\pi x} \geqq \frac{2}{\pi}
$$

for $|x| \leqq 1 / 2$, we have

$$
\begin{aligned}
& \int_{-\Delta}^{\Delta}|F(x)|^{2} d x \leqq \frac{\pi^{2}}{4} \int_{-\infty}^{\infty}|F(x)|^{2} \operatorname{sinc}^{2} \frac{x}{2 \Delta} d x \\
& \quad=\frac{\pi^{2} \Delta}{2} \int_{-\infty}^{\infty} \sum_{i} \sum_{j} a_{i} \bar{a}_{j} \mathrm{e}\left(2 \Delta\left(s_{i}-s_{j}\right) y\right) \operatorname{sinc}^{2} y d y=\frac{\pi^{2} \Delta}{2} S\left(\frac{1}{2 \Delta}\right),
\end{aligned}
$$

since $\Lambda(s)$ is the Fourier transform of $\operatorname{sinc}^{2} y$. For $X$ non-zero, we replace $a_{i}$ by $a_{i} \mathrm{e}\left(s_{i} X\right)$.

Our last lemma is geometrical.

Lemma 4 (Reciprocation). The lines $\alpha x+\beta y=1$ in the plane which do not go through the origin correspond one-to-one to the points $(\alpha, \beta)$ other than the origin, the poles of these lines with respect to the unit circle. If the line $\alpha x+\beta y=1$ is tangent to a given convex curve $C$ enclosing the origin, given in polar coordinates by $r=f(\theta)$, then the point $(\alpha, \beta)$ lies on a convex curve $D$ given parametrically by

$$
\alpha=\frac{\sin (\theta+\lambda)}{f(\theta) \sin \lambda}, \quad \beta=-\frac{\cos (\theta+\lambda)}{f(\theta) \sin \lambda},
$$

where $\lambda$ is the angle between the radius vector $O P$ and the tangent at the point $P$ with polar coordinates $r$ and $\theta$ on the original curve $C$, with

$$
\cot \lambda=\frac{d}{d \theta} \log f(\theta), \quad 0<\lambda<\pi
$$

The radius of curvature $\sigma$ at the point $Q$ on $D$ corresponding to the tangent to $C$ at $P$ is related to the radius of curvature $\rho$ of $C$ at $P$ by 


$$
\rho \sigma \sin ^{3} \lambda=1
$$

Proof. If $\alpha x+\beta y=1$ is the tanget to $C$ at the point $P(r \cos \theta, r \sin \theta)$, then

$$
\alpha \cos \theta+\beta \sin \theta=1 / r
$$

and the gradient at $P$ is

$$
\left(r \cos \theta+\frac{d r}{d \theta} \sin \theta\right) /\left(\frac{d r}{d \theta} \cos \theta-r \sin \theta\right)=\frac{\cos \theta+\cot \lambda \sin \theta}{\cot \lambda \cos \theta-\sin \theta}=\tan (\theta+\lambda)
$$

and asserted. Thus

$$
\begin{gathered}
-\alpha / \beta=\tan (\theta+\lambda), \\
\alpha \cos (\theta+\lambda)+\beta \sin (\theta+\lambda)=0 .
\end{gathered}
$$

The simultaneous equations (3) and (4) for $\alpha$ and $\beta$ give (1) of the theorem.

Differentiating, we get from (3)

$$
\begin{aligned}
\frac{d \alpha}{d \theta} & \cos \theta+\frac{d \beta}{d \theta} \sin \theta=-\frac{1}{r^{2}} \frac{d r}{d \theta}+\alpha \sin \theta-\beta \cos \theta \\
= & -\frac{\cot \lambda}{r}+\frac{\sin \theta \sin (\theta+\lambda)+\cos \theta \cos (\theta+\lambda)}{r \sin \lambda} \\
& =-\frac{\cos \lambda}{r \sin \lambda}+\frac{\cos \lambda}{r \sin \lambda}=0 .
\end{aligned}
$$

Thus

$$
\frac{d \beta}{d \alpha}=-\cot \theta=\tan \left(\theta+\frac{\pi}{2}\right)
$$

and the tangent to the curve $D$ at the point $Q$ is

$$
x r \cos \theta+y r \sin \theta=1,
$$

corresponding to the point $P$ on $C$; we have verified that reciprocation is a self-inverse transformation. Differentiation in (4) gives

$$
\begin{gathered}
\frac{d \alpha}{d \theta} \cos (\theta+\lambda)+\frac{d \beta}{d \theta} \sin (\theta+\lambda)=(\alpha \sin (\theta+\lambda)-\beta \cos (\theta+\lambda))\left(1+\frac{d \lambda}{d \theta}\right) \\
=\frac{1}{r \sin \lambda}\left(1+\frac{d \lambda}{d \theta}\right) .
\end{gathered}
$$


Solving (5) and (6) as simultaneous equations for $d \alpha / d \theta$ and $d \beta / d \theta$ gives

$$
\frac{d \alpha}{d \theta}=-\frac{\sin \theta}{r \sin ^{2} \lambda}\left(1+\frac{d \lambda}{d \theta}\right), \frac{d \beta}{d \theta}=\frac{\cos \theta}{r \sin ^{2} \lambda}\left(1+\frac{d \lambda}{d \theta}\right)
$$

Since $\theta+\pi / 2$ is the tangent angle, the radius of curvature of $D$ is given by

$$
\sigma=\frac{1}{r \sin ^{2} \lambda}\left(1+\frac{d \lambda}{d \theta}\right)
$$

The arc length of $C$ is given by

$$
\left(\frac{d s}{d \theta}\right)^{2}=r^{2}+\left(\frac{d r}{d \theta}\right)^{2}=r^{2} \operatorname{cosec}^{2} \lambda
$$

Since the tangent angle for $C$ is $\theta+\lambda$, we have

$$
\left(1+\frac{d \lambda}{d \theta}\right) \rho=\frac{d(\theta+\lambda)}{d \theta} \frac{d s}{d(\theta+\lambda)}=\frac{r}{\sin \lambda}
$$

We deduce (2) from (7) and (8).

We can now prove our theorem on the mean value of the discrepancy $D_{M}$ under enlargement of the curve $C_{M}$.

Proof of the Theorem. The result is not a Parseval inequality, so we must work to get convergence. From the mean-to-max argument (Lemma 2), we have

$$
\left|D_{u}\right| \leqq 5 A \delta u+\frac{\sqrt{ }(6 M)}{\delta}\left|\int_{u-\delta}^{u} \frac{D_{t}}{\sqrt{ } t} d t\right|+\frac{\sqrt{ }(6 M)}{\delta}\left|\int_{u}^{u+\delta} \frac{D_{t}}{\sqrt{ } t} d t\right|
$$

so that

$$
\int_{M}^{M+1}\left|D_{u}\right|^{2} d u \leqq 75 A^{2} \delta^{2}(M+1)^{2}+\frac{216 M}{\delta^{2}} \int_{M-\delta}^{M+1}\left|\int_{u}^{u+\delta} \frac{D_{t}}{\sqrt{ } t} d t\right|^{2} d u .
$$

We choose $\delta=1 / \sqrt{ } M$, so that the first term is $O(M)$. Next we write

with

$$
D_{t}=\sum_{(h, k) \neq(0,0)}(-2 i) \operatorname{Im} b_{t}(h, k)+O\left(\frac{1}{\sqrt{M}}\right)
$$




$$
\begin{gathered}
\int_{u}^{u+\delta} \frac{D_{t}}{\sqrt{t}} d t=-2 i \sum_{(h, k) \neq(0,0)} \operatorname{Im} \frac{\sqrt{\rho(k,-h)}}{2 \pi i\left(h^{2}+k^{2}\right)^{3 / 4}} \int_{u}^{u+\delta} \mathrm{e}\left(-t L(k,-h)+\frac{1}{8}\right) d t+O\left(\frac{\delta}{\sqrt{M}}\right) \\
=-2 i \sum_{(h, k) \neq(0,0)} \frac{\sqrt{\rho(k,-h)}}{\left(h^{2}+k^{2}\right)^{3 / 4}} \operatorname{Im} \mathrm{e}\left(-u L(k,-h)+\frac{1}{8}\right) \frac{1-e(-\delta L(k,-h))}{2 \pi i L(k,-h)}+O\left(\frac{\delta}{\sqrt{M}}\right) \\
=-2 i \sum_{(h, k) \neq(0,0)} \frac{\operatorname{Im} b_{u+\delta / 2}(h, k)}{\sqrt{(u+\delta / 2)}} \cdot \frac{\sin \delta \pi L(k,-h)}{\pi L(k,-h)}+O\left(\frac{\delta}{\sqrt{M}}\right),
\end{gathered}
$$

where $\rho(v)$ denotes the radius of curvature at the point where the vector $\mathbf{v}$ is the anticlockwise tangent.

Thus

$$
\int_{M}^{M+1}\left|D_{u}\right|^{2} d u \leqq \frac{1728 M^{2}}{\pi^{2}} \int_{M-\delta}^{M+1}\left|\sum_{(h, k) \neq(0,0)} b_{u+\delta / 2}(h, k) \operatorname{sinc} \delta L(k,-h)\right|^{2} \frac{d u}{u+\delta / 2}+O(M) .
$$

We use Lemma 3 with

$$
X=M+(1-\delta) / 2, \Delta=(1+\delta) / 2 \text {, }
$$

and the real numbers $s_{i}$ as $-L(\mathbf{v})$ indexed by the non-zero integer vectors $\mathbf{v}$. The corresponding coefficients are

$$
a_{i}=\frac{\sqrt{ } \rho(\mathbf{v})}{2 \pi i|\mathbf{v}|^{3 / 2}} \mathrm{e}\left(\frac{1}{8}\right) \operatorname{sinc} \delta L(\mathbf{v})
$$

The integral in (9) is

$$
\begin{aligned}
& \leqq \\
& \qquad \frac{\pi^{2}(1+\delta)}{4} \sum_{v} \sum_{w} \operatorname{sinc} \delta L(\mathbf{v}) \operatorname{sinc} \delta L(\mathbf{w}) \frac{\sqrt{ }(\rho(\mathbf{v}) \rho(\mathbf{w}))}{(|\mathbf{v}||\mathbf{w}|)^{3 / 4}} \\
& \quad \times \mathrm{e}\left(\left(M+\frac{1+\delta}{2}\right)(L(\mathbf{w})-L(\mathbf{v}))\right) \Lambda((1+\delta)(L(\mathbf{v})-L(\mathbf{w}))) .
\end{aligned}
$$

We want to estimate this sum in order of magnitude. Non-zero terms have

$$
|L(v)-L(w)| \leqq 1
$$

Since the origin is an interior point of $C$, we have

$$
L(v) \asymp|v|
$$


The inequality (11) introduces the non-uniformity in the position and orientation of $C$. We use it to deduce that when the $\Lambda$ function is non-zero, then

$$
|v| \asymp|w| .
$$

Hence the expression (10) is

$$
O\left(\sum_{\mathbf{v}} \frac{\operatorname{sinc}^{2} \delta L(\mathbf{v})}{|\mathbf{v}|^{3}} \sum_{\mathbf{w}} 1\right) .
$$

where the second sum is over w satisfying (11), and we have used Cauchy's inequality in the form

$$
\left|\frac{2 \operatorname{sinc} \delta L(\mathbf{v}) \operatorname{sinc} \delta L(\mathbf{w})}{(|\mathbf{v}||\mathbf{w}|)^{3 / 4}}\right| \leqq \frac{\operatorname{sinc}^{2} \delta L(\mathbf{v})}{|\mathbf{v}|^{3 / 2}}+\frac{\operatorname{sinc}^{2} \delta L(\mathbf{w})}{|\mathbf{w}|^{3 / 2}}
$$

to break the symmetry, interchanging $\mathbf{v}$ and $\mathbf{w}$ in the second sum.

To estimate the number of vectors $w$ satisfying (11), we consider the equation

$$
L(k,-h)=-\lambda \text {. }
$$

By reciprocation (Lemma 4), the point $(h / \lambda, h / \lambda)$ lies on a smooth curve $D$ reciprocal to $C$. If (11) holds, then $v$ and $w$ correspond to points $\left(h_{1}, k_{1}\right)$ on $\lambda_{1} D,\left(h_{2}, k_{2}\right)$ on $\lambda_{2} D$, with

$$
\lambda_{2}=\lambda_{1}+O(1), \lambda_{2} \asymp \lambda_{1} \asymp|\mathbf{v}| .
$$

The lattice point $\left(h_{2}, k_{2}\right)$ lies within a distance $O(1)$ of the curve $\lambda_{1} D$, whose length is $O(|\mathbf{v}|)$. Hence for fixed $\mathbf{v}$ there are $O(|\mathbf{v}|)$ solutions of (11).

We divide the sum in (13) into blocks of the form $R \leqq|v| \leqq 2 R$, where $R$ is a power of two. Thus the sum in (13) has size

$$
O\left(\sum_{R} \frac{R^{2}}{R^{3}} \min \left(1, \frac{1}{\delta^{2} R^{2}}\right) R\right)=O\left(\sum_{R \leqq \sqrt{ } M} 1+\sum_{R>\sqrt{ } M} \frac{M}{R^{2}}\right)=O(\log M) .
$$

The main result follows on substituting this bound into (9). The Corollary follows by the mean-to-max argument of Lemma 2 with

$$
\delta=\left|D_{M}\right| / 5 A M .
$$

\section{REFERENCES}

1. H. Chaix, Démonstration élémentaire d'un théorème van der Corput, C. R. Acad. Sci. Paris 275 (1972), A883-885. 
2. Y. Colin dE Verdière Nombre de points entiers dans une famille homothétique de domains de $R$, Ann. Sci. Éc. Norm. Sup. (4) 10 (1977), 559-576.

3. J. G. van der Corput, Über Gitterpunkte in der Ebene, Math. Ann. 81 (1920), 1-20.

4. C. S. HERZ, Fourier transforms related to convex sets, Ann. of Math. 75 (1962), 81-92.

5. C. S. Herz, On the number of lattice points in a convex set, Amer. J. Math. 84 (1962), 126-132.

6. E. Hlawka, Integrale auf konvexen Körpern, Monatsh. Math. 54 (1950), 1-36, 81-99.

7. M. N. Huxtey, The rational points close to a curve, Ann. Scuola Norm. Sup. Pisa (Cl. Sci.), (4) 21 (1994), 357-375.

8. M. N. Huxley, Area, Lattice Points, and Exponential Sums (London Math. Soc.), to appear.

9. D. G., Kendall, On the number of lattice points inside a random oval, Quarterly J. Math. (Oxford) 19 (1948), 1-26.

10. W. G. Nowak, On the average order of the lattice rest of a convex planar domain, Math. Proc. Cambridge Philos. Soc. 98 (1985), 1-4.

11. W. G. Nowak, An $\Omega$-estimate for the lattice rest of a convex planar domain, Proc. Royal Soc. Edinburgh 100A (1985), 295-299.

12. W. SIERPIŃSKI, Sur un probleme du calcul des fonctions asymptotiques, Prace Mat.-Fiz. 17 (1906), 77-118.

13. G. Voronol, Sur une fonction transcendente et ses applications à la sommation de quelques séries, Ann. École Norm. Sup (3) 21 (1904), 207-267, 459-533.

School of Mathematics

University of Wales College of Cardiff

23, Senghenydd Road

CARDiff CF2 4YH 